\title{
Native mitochondrial RNA-binding complexes in kinetoplastid RNA editing differ in guide RNA composition
}

\author{
BHASKARA R. MADINA, ${ }^{1}$ VIKAS KUMAR, ${ }^{1}$ RICHARD METZ, ${ }^{2}$ BLAINE H.M. MOOERS, ${ }^{3}$ RALF BUNDSCHUH, ${ }^{4,5}$ \\ and JORGE CRUZ-REYES ${ }^{1,5}$ \\ ${ }^{1}$ Department of Biochemistry and Biophysics, Texas A\&M University, College Station, Texas 77843, USA \\ ${ }^{2}$ Texas AgriLife Genomics and Bioinformatics Service, Texas A\&M University, College Station, Texas 77845, USA \\ ${ }^{3}$ Department of Biochemistry \& Molecular Biology, University of Oklahoma Health Sciences Center, Oklahoma City, Oklahoma 73104, USA \\ ${ }^{4}$ Department of Physics, Department of Chemistry \& Biochemistry, Division of Hematology, Center of RNA Biology, The Ohio State University, \\ Columbus, Ohio 43210-1117, USA
}

\begin{abstract}
Mitochondrial mRNAs in kinetoplastids require extensive U-insertion/deletion editing that progresses $3^{\prime}$-to- $5^{\prime}$ in small blocks, each directed by a guide RNA (gRNA), and exhibits substrate and developmental stage-specificity by unsolved mechanisms. Here, we address compositionally related factors, collectively known as the mitochondrial RNA-binding complex 1 (MRB1) or gRNAbinding complex (GRBC), that contain gRNA, have a dynamic protein composition, and transiently associate with several mitochondrial factors including RNA editing core complexes (RECC) and ribosomes. MRB1 controls editing by still unknown mechanisms. We performed the first next-generation sequencing study of native subcomplexes of MRB1, immunoselected via either RNA helicase 2 (REH2), that binds RNA and associates with unwinding activity, or MRB3010, that affects an early editing step. The particles contain either REH2 or MRB3010 but share the core GAP1 and other proteins detected by RNA photo-crosslinking. Analyses of the first editing blocks indicate an enrichment of several initiating gRNAs in the MRB3010purified complex. Our data also indicate fast evolution of mRNA $3^{\prime}$ ends and strain-specific alternative $3^{\prime}$ editing within $3^{\prime}$ UTR or C-terminal protein-coding sequence that could impact mitochondrial physiology. Moreover, we found robust specific copurification of edited and pre-edited mRNAs, suggesting that these particles may bind both mRNA and gRNA editing substrates. We propose that multiple subcomplexes of MRB1 with different RNA/protein composition serve as a scaffold for specific assembly of editing substrates and RECC, thereby forming the editing holoenzyme. The MRB3010-subcomplex may promote early editing through its preferential recruitment of initiating gRNAs.
\end{abstract}

Keywords: RNA editing; deep RNA sequencing; guide RNA; mitochondrial RNA-binding complex MRB1; Trypanosoma brucei

\section{INTRODUCTION}

Kinetoplastid protozoa include medically relevant species of Trypanosoma and Leishmania with life cycle stages that experience dramatic adaptations to host and changes in energy metabolism (Vickerman 1985). A unique mitochondrial process of RNA editing by uridylate insertion and deletion is directed by guide RNAs (gRNAs) (Blum et al. 1990; Blum and Simpson 1990) and controlled in mRNA-specific and stagespecific manners by mechanisms that remain fundamentally unresolved (Stuart et al. 1997). The mitochondrial genome of Trypanosoma brucei (kinetoplast or kDNA) consists of several copies of a maxi-circle $(\sim 23 \mathrm{~kb})$ and thousands of minicircles $(\sim 1 \mathrm{~kb})$. Twelve out of 18 mRNAs encoded by the maxi-circle are edited. Minicircles encode most gRNAs, esti-

\section{${ }^{5}$ Corresponding authors}

E-mail cruzrey@tamu.edu

E-mail bundschuh@mps.ohio-state.edu

Article published online ahead of print. Article and publication date are at http://www.rnajournal.org/cgi/doi/10.1261/rna.044495.114. mated at $\sim 1200$ on their "sense" strand (Simpson et al. 2000; Ochsenreiter et al. 2007). gRNAs are primary transcripts with three regions: a short $5^{\prime}$ anchor that anneals to pre-edited or edited mRNA next to the sequence to be edited, a guide sequence with complementarity to edited mRNA (editing block), and a $\sim 10-15 \mathrm{U} 3^{\prime}$ tail added post-transcriptionally. An mRNA editing domain usually spans a set of juxtaposing and often overlapping editing blocks.

While the basics of the editing reaction catalyzed by RNA editing core complexes (RECCs) is well characterized, the regulatory aspects remain largely unknown (Madison-Antenucci et al. 2002; Simpson et al. 2004; Carnes and Stuart 2007; Cruz-Reyes and Read 2013). Editing domains in mRNAs mature from $3^{\prime}$ to $5^{\prime}$ in small blocks, each directed

\footnotetext{
(C) 2014 Madina et al. This article is distributed exclusively by the RNA Society for the first 12 months after the full-issue publication date (see http://rnajournal.cshlp.org/site/misc/terms.xhtml). After 12 months, it is available under a Creative Commons License (Attribution-NonCommercial 4.0 International), as described at http://creativecommons.org/licenses/ by-nc/4.0/.
} 
by a gRNA. Most mRNAs are extensively edited, while a few require limited editing or are never edited (Stuart et al. 1997). How a functional cognate substrate reaches the RECC is currently unknown. Accessory factors may facilitate selective binding of editing substrates, annealing, unwinding, and chaperone activities to stabilize precise base-pairing between the target mRNA and the gRNAs directing the U-insertions and U-deletions. Consistent with this idea, purified RECC was reported to be RNA-free and lacks the processivity and substrate specificity of editing in vivo (Rusche et al. 1997; Cruz-Reyes et al. 2001; Cifuentes-Rojas et al. 2005; Alatortsev et al. 2008; Carnes et al. 2011). Many non-RECC proteins impact editing (Aphasizhev and Aphasizheva 2011; Carnes et al. 2011; Cruz-Reyes and Read 2013; Hashimi et al. 2013). Some proteins preferentially affect a few mRNAs, while others affect a broader range. In a few cases, effects at initiation or during progression have been proposed, and RNA binding, annealing, and unwinding have been observed with recombinant protein or purified complexes (Ammerman et al. 2010; Hernandez et al. 2010; Kafkova et al. 2012). So, while much progress has been made, central long-standing questions remain perplexing, including the mechanisms of substrate recruitment, regulation of editing activity, and editing integration into mitochondrial RNA metabolism. The answer may involve a group of ribonucleoprotein particles of surprising complexity, known as the mitochondrial RNA-binding complex 1 (MRB1) (or gRNA binding complex, GRBC), which contain gRNA and a dynamic protein composition (Panigrahi et al. 2008; Weng et al. 2008; Hashimi et al. 2009; Hernandez et al. 2010; Ammerman et al. 2011). Subcomplexes of MRB1 (called here MRBs for simplicity) transiently associate via RNA linkers with several factors including RECCs, proteins that may affect processing and stability, and ribosomes. Our lab and others have proposed that these complexes may serve as "organizers" in the control of editing and its integration in mitochondria (Hernandez et al. 2010; Aphasizhev and Aphasizheva 2011; Cruz-Reyes and Read 2013; Hashimi et al. 2013). For example, the MRB3010 subunit (3010) affects an early editing step, whereas TbRGG2 impacts progression between blocks (Ammerman et al. 2010, 2011). We reported a helicase REH2 (H2) in an MRB that contains $3^{\prime}-5^{\prime}$ unwinding activity. Copurification of REH 2 with gRNA and unwinding activity is inhibited by mutation of its helicase or RNA binding domains (Hernandez et al. 2010). Importantly, RNAi-based repression of REH2 inhibits cell growth and RNA editing in trypanosomes (Hashimi et al. 2009; Hernandez et al. 2010; Madina et al. 2011).

We considered that MRBs of different protein composition could exhibit differences in their associated RNAs. We tested this hypothesis by performing the first next-generation sequencing (NGS) study of two native MRBs that contain either $\mathrm{H} 2$ or 3010 . Analyses of the first editing blocks showed that the 3010-associated MRB is enriched in initiating gRNAs. We also found specific copurification of MRBs with mRNAs that undergo editing. Interestingly, we identified differences in gRNA expression, including alternative editing of mRNA $3^{\prime}$ ends, between the Lister strain 427 used here, EATRO 164 cells in a recent NGS study of total gRNA in procyclics (Koslowsky et al. 2013), and currently annotated sequences. This adds a level of complexity to the potential of alternative editing first observed by the Hajduk lab (Ochsenreiter et al. 2008). Based on cumulative observations in our lab and others, we propose a model whereby subcomplexes of MRB1 with distinct RNA/ protein composition serve as scaffolds that recruit editing substrates and route them into transiently associated RECCs. A dynamic higher-order MRB1 complex, formed by several subcomplexes with specialized roles, may provide the necessary context for concerted substrate selection or usage during editing initiation and progression.

\section{RESULTS}

\section{Native subcomplexes of MRB1 containing either REH2 or MRB3010}

MRB1 ribonucleoprotein complexes were previously isolated from procyclic trypanosomes expressing tagged copies of REH2 or MRB3010 (3010), and their protein composition examined in mass spectrometric studies (Hernandez et al. 2010; Ammerman et al. 2011), but the RNA composition of these complexes is not known. Furthermore, the large tag in the constructs or ectopic expression could induce unintended effects on the protein and RNA composition of MRBs. The current study characterized immunoprecipitated (IP) native MRBs from mitochondrial extracts using affinitypurified peptide polysera against $\mathrm{H} 2$ or 3010 (Fig. 1A; Supplemental Fig. S1). Helicase $\mathrm{H} 2$ is often partially fragmented in extracts (Hernandez et al. 2010), whereas 3010 migrates slightly below IgG on SDS-PAGE. Interestingly, 3010 was not detected in Western analyses of native H2 IPs, whereas $\mathrm{H} 2$ is not detected or is barely visible in native 3010 IPs. We reproduced this observation multiple times using mitochondrial extract or whole-cell lysate in the IPs (e.g., Supplemental Fig. S2). Consistent with our analysis of native MRBs, $\mathrm{H} 2$ was not detected in a previous affinity-purification of tagged MRB3010 (Ammerman et al. 2011). So, loss of H2 in that study was not caused by the large tag in 3010 but instead is an intrinsic feature of native 3010-MRB.

A transient association between RECC and H2-purified MRB was previously observed (Hernandez et al. 2010). RECC subunits were almost undetectable with different antibodies in the current native $\mathrm{H} 2$ and 3010 purifications (Fig. 1B; data not shown). However, editing ligases can be radiolabeled by a sensitive auto-adenylation assay indicating substoichiometric levels of RECC (data not shown; Hernandez et al. 2010). As expected, both natively purified MRBs contain gRNA (Fig. 1C) and the proposed core GAP1 subunit (Fig. 1D), consistent with previous purifications of REH2 and MRB3010 (Hernandez et al. 2010; Ammerman et al. 2011). 


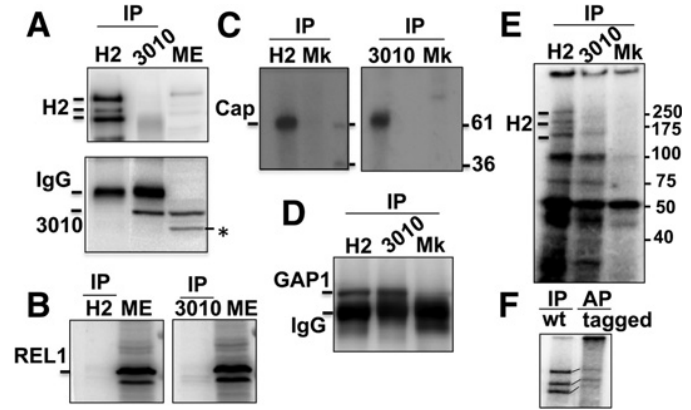

FIGURE 1. Native immunopurified subcomplexes of MRB1 with either $\mathrm{H} 2$ or 3010 subunits. (A) Western analysis of $\mathrm{H} 2$ and 3010 in IPs by the indicated antibodies, and in mitochondrial extract (ME). H2 $(\sim 240 \mathrm{kDa})$ is often fragmented, and $3010(57.5 \mathrm{kDa})$ migrates slightly below IgG in the IPs. A fainter band below 3010 is a breakage product of this protein in $\mathrm{ME}\left({ }^{*}\right)$. The blot was split into halves treated with either anti-H2 or anti-3010 antibodies. (B) Western analysis of the RECC subunit REL1 ligase in IPs and ME. $(C)\left[{ }^{32} \mathrm{P}\right] \mathrm{G}$-capping of gRNA $5^{\prime}$ triphosphate with guanylyltransferase on $15 \%$ UREA-PAGE to concentrate gRNA in a discrete band. $(D)$ Western analysis of the MRB1 subunit GAP1 in test and mock IPs. Mock IPs (Mk) used an irrelevant affinity-purified antibody. (E) Site-specific crosslinking of H2 and 3010 IPs with a pre-edited mRNA construct whose first editing site contains ${ }^{32} \mathrm{P}$ in its phosphodiester bond and 4-thioU in the flanking $5^{\prime}$ nucleotide. After RNase trimming, the protein-RNA adducts were resolved on $10 \%$ SDS-PAGE. ( $F$ ) Crosslinks as in $E$, but on a high-resolution $6 \%$ SDS-PAGE. Native (wt) and ectopic (tagged) $\mathrm{H} 2$ differ in mobility due to an $\sim 20 \mathrm{kDa}$ tag. Tagged $\mathrm{H} 2$ was affinity-purified (AP). Intact and fragmented H2 are marked. Molecular markers are in kDa. All IPs (200 $\mathrm{mM} \mathrm{KCl}, 5 \% \mathrm{BSA}$ ) used precleared extract.

Only endogenous gRNA has been reported to bind purified MRB1 complexes; however, several subunits have domains that suggest a role in RNA biology (Panigrahi et al. 2008; Weng et al. 2008). For example, our lab reported that $\mathrm{H} 2$ crosslinks with an mRNA editing site bearing a photo-reactive group (Hernandez et al. 2010). Importantly, immunopurified $\mathrm{H} 2$ and 3010 MRBs produced similar crosslinking patterns with this substrate (Fig. 1E), except for the crosslinks induced by $\mathrm{H} 2$ itself. The $\mathrm{H} 2$ crosslinks were confirmed by their mobility shift in a direct comparison of tagged vs. native $\mathrm{H} 2$ (Fig. 1F). This is the first time purified MRBs are directly compared by crosslinking, and the analysis suggests that $\mathrm{H} 2$ and 3010 MRBs share several RNA-binding proteins. Most of these crosslinks were not detected in the mock IP. Together, these data describe two native subcomplexes of MRB1 that stably copurify with gRNA, the core GAP1 subunit, and several common RNA-crosslinking proteins. However, these MRBs were readily distinguished by the presence of $\mathrm{H} 2$ or 3010. This result prompted us to investigate the RNA composition of our purified samples.

\section{Native H2-MRB and 3010-MRB subcomplexes differ in gRNA composition}

To address the possibility that MRBs of different protein composition may also differ in their gRNA complement, we compared the gRNA pools from H2, 3010, and mock IPs, and from procyclic (PF) parasites using next-generation sequencing. Barcoded Illumina libraries made in parallel with comparable amounts of gRNA scored in a capping assay (see Supplemental Fig. S3 and the Materials and Methods section) were multiplexed, and reads were aligned to edited mRNA sequences deposited at the KISS bioinformatics site (http://splicer.unibe.ch/kiss/) (Ochsenreiter et al. 2007). We filtered out fragmented RNA by scoring only gRNAs bearing a $3^{\prime}$ U-tail. Furthermore, because 3010 may impact editing at an early step (Ammerman et al. 2011), we focused on the first editing blocks of mRNAs (Fig. 2). In line with a recent characterization of the gRNA pool in PF cells (Koslowsky et al. 2013), we found that initiating gRNAs for several mRNAs occur in relatively low abundance. Because our libraries used limiting gRNA amounts extracted from the IPs, we only found initiating gRNAs for six mRNAs. The following analyses compare cumulative nucleotide frequency plots (NFPs) of edited mRNA coverage (Fig. 2) and individual frequency values and the ratio of major gRNAs in editing blocks 1 and 2 (B1 and B2, respectively) (Table 1). Some gRNAs predicted alternative editing sequences to those currently annotated (termed B1.alt or B2.alt) and are further discussed in Figure 3. Negligible gRNA levels in the mock IP library confirmed the specificity of our MRB libraries (Table 1).

\section{Cytochrome $B(\mathrm{CY} b)$}

mRNA CYb has a small editing domain ( $\sim 50 \mathrm{nt})$ with only two blocks (Fig. 2A). Notably, the coverage of the B1 and B2 blocks was similar in the parasites but different between MRBs. Relative to the B2 gRNA in each library, the initiating B1 gRNA appears enriched in 3010-MRB. A higher coverage of the $\mathrm{B} 2$ editing block in the H2-MRB libraries relative to the B1 block also suggests intrinsic differences between native MRBs. Table 1 shows the actual values and ratio of major gRNAs in these blocks (B1/B2), which is a simple parameter to evaluate relative B1 enrichment in MRBs independent of loading.

\section{NADH dehydrogenase subunits 7 and 8 (ND7 and ND8)}

mRNA ND7 has two separate editing domains with initiating gRNAs that were enriched in 3010-MRB (Fig. 2B,C). Interestingly, the $5^{\prime}$ domain has two possible initiating gRNAs, whose guide sequences have different $5^{\prime}$ ends but the same $3^{\prime}$ end: B1a and B1b (Fig. 2B). The relative values of B1b and B2 gRNAs suggested a preferential binding of $\mathrm{B} 1 \mathrm{~b}$ gRNA in 3010-MRB. In contrast, the Bla gRNA was too low in the MRB libraries and absent in the PF library (Fig. 2B; Table 1). As in mRNA CYb, the H2-MRB library exhibited a higher coverage of the $\mathrm{B} 2$ gRNA for the $5^{\prime}$ domain. In the ND7 3' domain (Fig. 2C), the B1 gRNA was dramatically increased in 3010-MRB. In contrast, the B2 gRNA in this domain was too low to quantify in the MRB libraries. In mRNA ND8 (Fig. 2D), we found an initiating gRNA but 


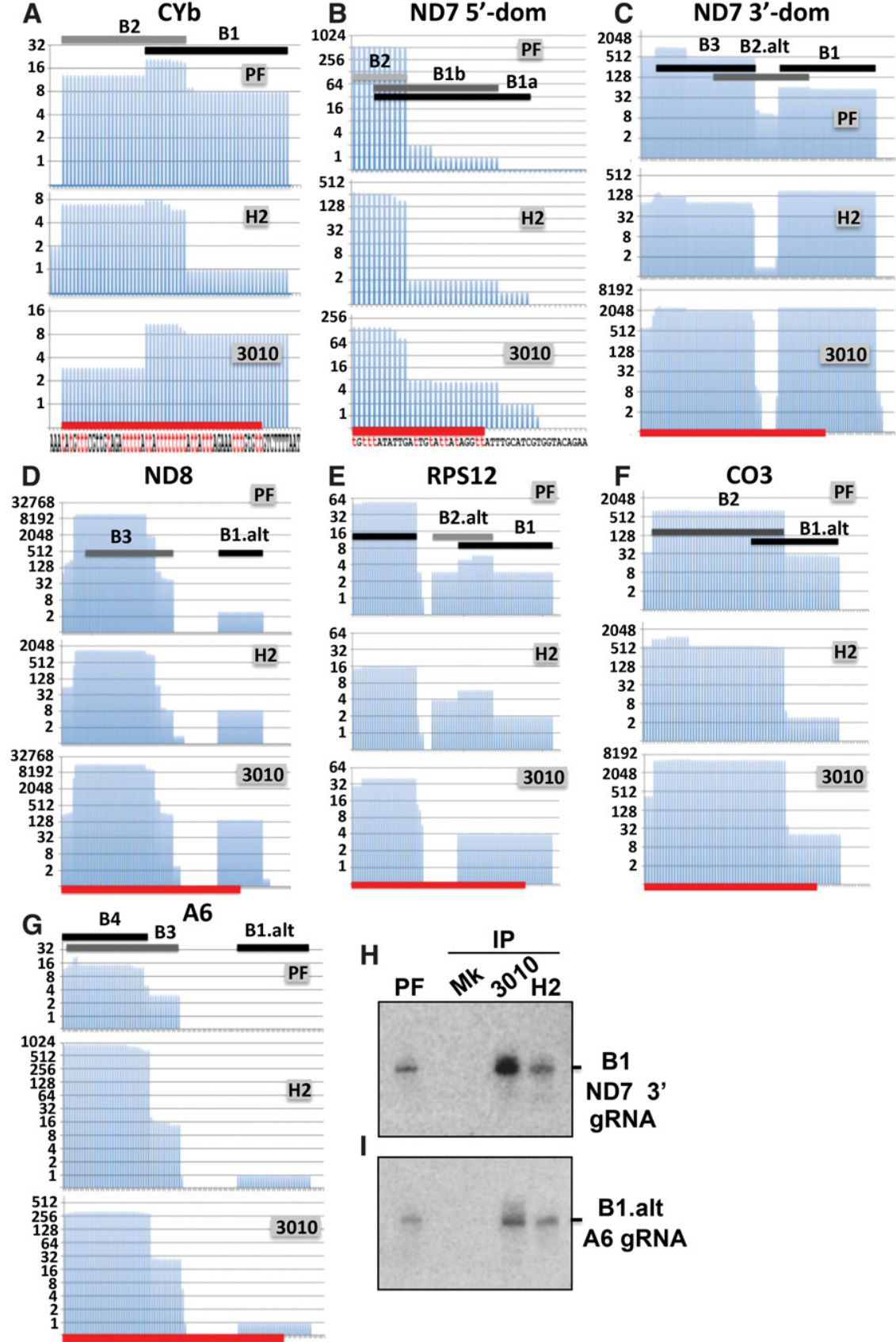

FIGURE 2. Analysis of edited mRNA coverage by gRNAs in Illumina libraries. (A-G) Steadystate gRNA from PF parasites and gRNA in immunopurified MRBs are annotated in blocks of edited sequence, each directed by a gRNA. Cumulative standard and G.U pairs between edited mRNA and gRNAs are scored in nucleotide frequency plots (NFPs $\left.\log _{2}\right)$, including initiating gRNAs (i.e., block 1 or B1) and major upstream gRNAs (B2 or B3) in our libraries. Some gRNAs may guide alternative editing (e.g., B1.alt and B2.alt), and the ND7 5' domain uses at least two similar initiating gRNAs (B1a and B1b). The entire editing domain in cytochrome B (CYb) and the $3^{\prime}$ terminus of other domains are plotted. The sequence of $\mathrm{CYb}$ (including all $34 \mathrm{U}$-insertions as lowercase t's) and the ND7 5' domain are shown. Equal protein loads were applied to the IPs, and the gRNA was gel-isolated and extracted. gRNA from IPs and total RNA were adjusted to apply comparable amounts in the libraries (e.g., Supplemental Fig. S3; see Materials and Methods). gRNA from the IPs was directly ligated to the adapters, whereas total gRNA from PF parasites was first treated with $5^{\prime}$ monophosphate specific Terminator exonuclease, which degrades rRNA but not $5^{\prime}$ triphosphate gRNA ends. $(H, I)$ Northern blots of select initiating gRNAs from IPs and PF cells. The blot was hybridized with the A6 gRNA probe, stripped, and re-used with the ND7 gRNA probe. 15\% UREA-PAGE was run as in Figure 1C. not a B2 gRNA in the libraries. However, a relatively high value of the initiating gRNA in 3010-MRB when compared to upstream gRNAs also suggested a specific increased abundance in this complex. B1/B2 determinations assuming a value of $\leq 1$ for nondetected gRNAs indicated B1 enrichment in 3010-MRB for ND8 and both ND7 domains, especially in the $3^{\prime}$ domain.

Ribosomal protein subunit 12 (RPS12), cytochrome oxidase 3 (CO3), and ATPase subunit 6 (A6)

Our libraries contain possible initiating gRNA for all three mRNAs RPS12, $\mathrm{CO} 3$, and A6 (Fig. 2E-G), whereas a B2 gRNA was missing for A6. In mRNA $\mathrm{CO} 3$, both $\mathrm{B} 1$ and $\mathrm{B} 2$ gRNAs were increased in 3010-MRB. However, the B1/ B2 ratio for mRNA RPS12 suggested a preferential increase of $\mathrm{B} 1$ gRNA in 3010-MRB (Table 1). In A6 mRNA, we found an initiating gRNA in the MRB libraries but no B2 gRNA in any of the libraries. Nevertheless, the B1 gRNA was too low in our libraries to be examined quantitatively.

As a complementary approach to our above NGS studies, we performed Northern blots of B1 gRNAs for the ND7 $3^{\prime}$ domain and A6 mRNA from direct IP samples of MRBs (Fig. 2H,I). Consistent with our findings in the libraries, the level of B1 gRNA in the ND7 $3^{\prime}$ domain was much higher in the 3010 IP than in the H2 IP. The B1.alt gRNA in A6 mRNA was slightly higher in the 3010 than in the H2 IP, possibly reflecting a moderate but reproducible difference in capping signal in our unadjusted samples from direct IPs (see Supplemental Fig. S3 and the preparation of libraries in the Materials and Methods section).

Collectively, these data showed important differences between our MRB libraries in coverage NFPs of the first editing blocks. Relative comparisons of gRNAs $(\mathrm{B} 1 / \mathrm{B} 2)$ in the same library were consistent with an enhanced association of initiating gRNAs for mRNAs CYb, ND7 (both domains), ND8, and RPS12 in 3010-MRB. Furthermore, evidently 
TABLE 1. Relative amounts of $B 1$ and $B 2$ gRNAs

\begin{tabular}{|c|c|c|c|c|c|c|c|c|c|c|}
\hline & \multicolumn{4}{|c|}{ B1 } & \multicolumn{4}{|c|}{ B2 } & \multicolumn{2}{|c|}{ B1/B2 } \\
\hline & PF & H2-IP & 3010-IP & Mock-IP & PF & $\mathrm{H} 2-\mathrm{IP}$ & 3010-IP & Mock-IP & H2-IP & 3010-IP \\
\hline $\mathrm{CYb}$ & 8 & 1 & 8 & nd & 10 & 5 & 3 & 1 & 0.2 & 2.7 \\
\hline ND7 5' dom & $1^{* *}$ & $1^{* *}$ & $5^{* *}$ & nd & 494 & 180 & 82 & 8 & 0.006 & 0.06 \\
\hline ND7 3' dom & 60 & 179 & 2592 & 9 & 8 & 1 & nd & nd & 179 & $\geq 2592$ \\
\hline ND8 & 3 & 9 & 147 & nd & nd & nd & nd & nd & $\geq 9$ & $\geq 147$ \\
\hline RPS12 & 3 & 2 & 4 & nd & 3 & 4 & nd & 3 & 0.5 & $\geq 4$ \\
\hline CO3 & 28 & 3 & 21 & nd & 801 & 565 & 5208 & 22 & 0.005 & 0.004 \\
\hline A6 & 0 & 1 & 1 & nd & nd & nd & nd & nd & $\geq 1$ & $\geq 1$ \\
\hline ND7 5' dom & $0^{*}$ & 1 & $1 *$ & nd & & & & & & \\
\hline
\end{tabular}

Frequency of a gRNA is defined as the value of the dominant $5^{\prime}$ end in a block. The $5^{\prime}$ end of guide sequences is generally more conserved than the $3^{\prime}$ end. $\left(^{*}\right)$ and $\left(^{* *}\right)$ distinguish two analog initiating gRNAs (B1a and B1b, respectively) with a different $5^{\prime}$ end but the same $3^{\prime}$ end. A block may include one gRNA or multiple redundant gRNAs of equivalent guide sequence with conserved nucleotide polymorphisms. Redundant gRNAs may reflect genetic drift of a minicircle or homolog genes in different minicircles. (nd) Not detected. Calculations of B1/B2 assume "nd" to be $\leq 1$.

different patterns in the coverage NFPs between procyclic and MRB libraries also indicated differential gRNA binding by MRBs. An increased selectivity of B1 gRNA by 3010MRB implies that this complex may be more active in editing initiation than H2-MRB. Interestingly, the mRNAs ND7 ( $3^{\prime}$ domain) and ND8 exhibited the largest relative accumulation of initiating gRNA.

\section{Alternative editing at mRNA $3^{\prime}$ ends and gRNA divergence in $T$. brucei strains}

Some gRNAs in Figure 2 suggest alternative editing patterns compared to annotated edited mRNAs from studies in the early 1990s. All cases of alternative editing discussed here significantly extend the length and quality of the predicted duplex between guide sequence and edited mRNA. Importantly, the observed mismatches with annotated sequence were outside the gRNA anchor. Some guide sequences were well conserved between Lister 427 studied here and EATRO 164 cells in a recent study by Koslowsky et al. (Fig. 3 ), but others exhibited important strain-specific differences, consistent with a rapid evolution of minicircles. This section compares annotated and predicted alternative editing directed by B1 and B2 gRNAs in our libraries and examines the conservation of guide sequences between strains. Some gRNAs with very similar or identical guide sequences in both strains often exhibit highly diverged nonguiding termini.

\section{CYb editing domain}

The B1 and B2 gRNAs in Lister 427 were identical to those reported by Koslowsky et al., including a $5^{\prime}$ terminal run of As (Fig. 3A). As indicated by that lab, an initiating gRNA, $\mathrm{gCYb}[560 \mathrm{~A}]$ from an earlier study in EATRO 164 cells (Koslowsky et al. 1992) has the same guide sequence but a very different $5^{\prime}$ end. This gRNA was not present in their recent library from EATRO 164 cells or ours reported here.

\section{ND7 3' editing domain}

The guide sequence of the B2.alt gRNA formed one mismatch (Fig. 3B[1]). However, simple alternative editing (one U-insertion and one U-deletion) would allow annealing with most of the gRNA (Fig. 3B[2]). Notably, this would also substitute two of the three $\mathrm{C}$-terminal amino acids without changing the coding frame. The guiding sequence of B2.alt is nearly identical in Lister 427 and EATRO 164 cells (Fig. $3 \mathrm{~B}[3])$.

\section{ND7 5 ' editing domain}

As mentioned above, this domain had two initiating gRNAs (B1a and B1b) (Fig. 3C[1]). The Bla gRNA was not found in the EATRO 164 library, but the B1b gRNA was the same in Lister 427 and EATRO 164 cells. An additional initiating gRNA, gND7(147-199), in EATRO 164 cells was not present in our libraries. These three gRNAs shared significant homology (Fig. 3C[2]) including a 7-nt identity at their $5^{\prime}$ termini. Based on this $5^{\prime}$ conservation, Koslowsky et al. suggests that gND7(147-199) could form an alternative anchor duplex (i.e., the same anchor by the Bla gRNA). However, the resulting alternative editing would introduce two A:C mismatches, decreasing the quality of the duplex (Koslowsky et al. 2013).

\section{CO3 editing domain}

The B1.alt gRNA in our libraries (Fig. 3D[1]) was not seen in the EATRO 164 library, and vice versa, we did not find the initiating gRNA in Koslowsky's study. Alternative editing by the B1.alt gRNA would substantially modify the $3^{\prime}$ UTR sequence (Fig. 3D[2]). Coincidently, both the B1.alt gRNA 
Downloaded from rnajournal.cshlp.org on April 26, 2023 - Published by Cold Spring Harbor Laboratory Press

Differential gRNA partition in MRB subcomplexes

A Cyb

TAAATTTTATATAAAtAtGtttCGttGtAGAtttttAttAttttttttAttAtttAGAAAtttGtGttGTCTTTTAATGTC

7 TATCAATAGGAGGGGTAATGAGTCTTTAGATGTAACAGGAAAAAAAAAA gCYb(54-91) B1

6 TATAAGAGTCGTATAGgGTGGTATTTGAGAGTAATAAAAAAAAA $\mathrm{gCYb}(32-64)$ B2

B ND7 3' editing domain $\quad \mathrm{V} D \mathrm{D}$

ttGCCGttttAGGtAAtAttGAtGttGtttttGGAtCC $\overline{G T A} \frac{\mathrm{D} A T C G t}{t}$ AGttttAtAtGtGAGGTTATTGt AGGATTGTTTAAAATTGAATAAAAA

(1)

7 TAATTTAGTGATTAGAATATGTACTTCAGTGACATCCTAACAAATATA gND7(1269-1320) B1

10 TTTTATTATAGTTACAATGAGGGTCTAGGCTINTAGCAATCAAATATATA gND7(1240-1268) B2.alt

Alternative editing by B2.alt $\quad \mathrm{E} \underline{\mathrm{Y}} \mathrm{R}$

ttGCCGttttAGGtAAtAt tGAtGttGtttttGGAtCcG $\star^{\star A G t A T C G t} t$ AGttttAtAtGtGAGGTTATTGtAGGATTGTTTAAAATTGAATAAAAA

(2) 10 TTTTATTATAGTTACAATGAGGGTCTAGGC-TTNTAGCAATCAAATATATA gND7(1240-1268) B2.alt

5'ATATATAAACTAACGATNTTGGATCTGGGAGTAACATTGATATTATTTT T1 10 gND7(1240-1268) B2.alt

(3) 5 AAACTAACGATATTCGGATCTGAGAGTAACATTGATATTATTMT T 7 gND7(1240-1268) in Koslowsky et al

C ND7 5' editing domain

AtGttGtttattGtAtttttGtGGtGAAtttattGtttaTATTGAtTGtAttAtAGGttATTTGCATCGTGGTACAGAAAAG

(1)

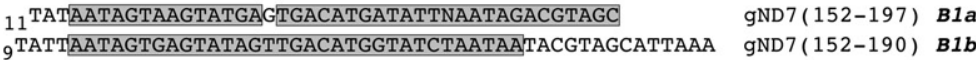

10 TTAATAGATAGTATGAAGACGTCACTTAGATGACAAATATAACATATA gND7(124-168) B2

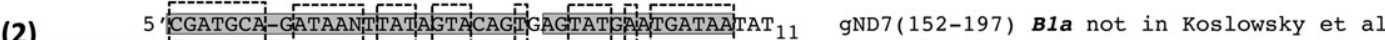

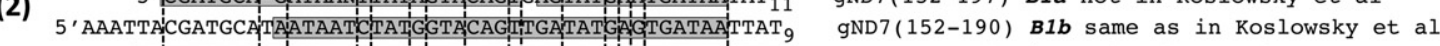
5' ATATATACACGATGCA-GATAATCPATAGTATGATTGATATAMGITGATAATTTT 9 gND7(147-199) in Koslowsky et al

D $\mathrm{CO} 3$

GtAtttGtGtGtG $\underline{\underline{\underline{t a A}}}$ ttttAttGGtGttttTTPAGTGttGAttAGttAAtttGtAttGGTAGTTTGTAGGAAGTT

13 TAATATAAGATCTGCAGGTAGCTAATTAATTAAACATAACTATAAAAAA gCO3(972-996) B1.alt

6TTAAATGTACATGTTAGAGTAACTATAGAAAAGTCAACAACTAAATATG gCO3(935-977) B2

(1) Alternative editing by B1.alt

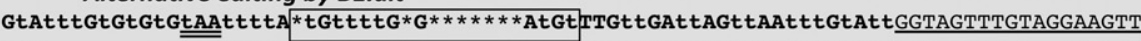

(2) 15 TAAT-ATAAGAT-C-------TGCAGGTAGCTAATTAATTAAACATAACTATAAAAAA gCO3(951-996) B1.alt

E ND8

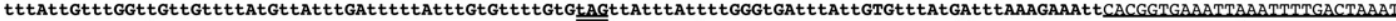

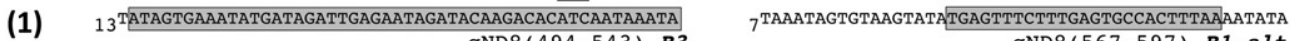

Alternative editing by B1.alt gND8 (494-543) B3 gND8 (567-597) B1.alt

GtAGttatt tAttttGGGtGAtt tAttGTGtttatGEAttttAAAGAAAt tCACGGTGAAATTAAATTTTGACTAAAT

(2) 6 TAAATAGTGTAAGTATATGAGTTTCTTTGAGTGCCACTTTAAAATATA gND8(538-597) B1.alt

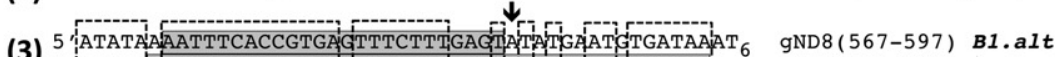

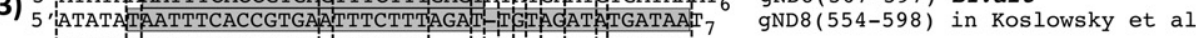

F RPS12

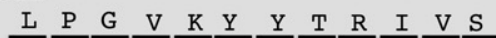

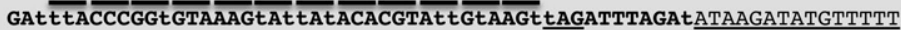

(1) 10 TAAGTGTATAATGTTCAATCTAAGTCTATATTCTATACAATAT gRPS12(309-349) B1

6TGAGTAACTAGATGTGTCATGATATGTGCATAACATTAATATA gRPS12(294-323) B2.alt

$\stackrel{L}{P} \quad \mathbf{R} \quad \mathrm{W} \quad \mathrm{F} I \quad$ Alternative editing by B2.alt

GAtttACCtCGttG*GttTAtAtAGtAttAtACACGTAttGtAAGttAGATTTAGAtATAAGATATGTTTTT

(2) 6TGAGTAAC-TAGATGTGTCATGATATGTGCATAACATTAATATA gRPS12(283-322) B2.alt

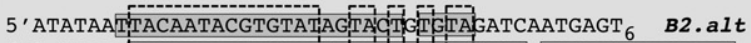

(3) 5'ATATACAATACGTGTATGATAMTTMTATACTGGATAGATCAGTGAAAT 13 gRPS12(267-322) in Koslowsky et al

G A6

GGAttttttGttGtttttGttGtttGtttAGttttGtAtttGAtttttGAtAGttAttAtAttGttGttGAAAtttGGttTGttATTGGAGTTATAGAATAB

13 TTAATAGTATGGTGAC-ATTTTGACINAAGCAGTAACCTCAATATCA gA6(774-822) B1.alt

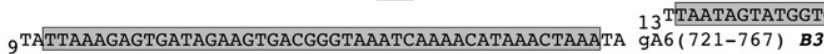

(1) Alternative editing by B1.alt

tGAtttttGAtAGttAttAtAttGttG*tGAAAtt*GGtttTGttATTGGAGTTATAGAATAA

(2) 13 TAATAGTATGGTGAC-ATTTTGA-CTNAAGCAGTAACCTCAATATCA gA6(774-822) B1.alt

(3) 5 , AूTATAACTCCAATGACGANTCAGTTTACAGTGHATGATAAT 13 gA6(774-822) B1.alt

5' ATACTATAACTCCAATGACGAAATCAGTTTTACAGTGATATGATAA 13 gA6(774-822) in Koslowsky et al

FIGURE 3. (Legend on next page) 
and both $\mathrm{CYb}$ gRNAs contain a $5^{\prime}$ A-run. The B2 gRNA, gCO3(935-977), was the same in both strains.

\section{ND8 editing domain}

The B1.alt gRNA formed a relatively short continuous duplex with annotated edited sequence (Fig. 3E[1]). However, alternative editing by a single U-insertion in the $3^{\prime}$ UTR extended the duplex dramatically (Fig. 3E[2]). This gRNA was similar to gND8(554-598) in EATRO 164 cells, except that the B1.alt gRNA in Lister 427 cells had one additional internal guiding "A" (see arrow, Fig. 3E[3]) that causes the alternative editing.

\section{RPS12 editing domain}

The guide sequence of the B1 gRNA (Fig. 3F[1]) was the same in Lister 427 and EATRO 164 cells. Alternative editing by the B2.alt gRNA would shorten the encoded $\mathrm{C}$ terminus and substitute its last four amino acids (Fig. 3F[2]). The B2.alt gRNA was not seen in the EATRO 164 library, and we did not find the gRPS12(267-322) reported in the study by Koslowsky et al. (2013). However, these two gRNAs exhibit partial homology and have identical $5^{\prime}$ anchor sequences (Fig. 3F [3]). Interestingly, gRPS12(267-322) in EATRO 164 introduces an internal A:C mismatch that does not predict alternative edits (this " $\mathrm{A}$ " is noted by an arrow).

\section{A6 editing domain}

The B1.alt gRNA, gA6(774-822), formed two mismatches with annotated edited mRNA (Fig. 3G[1]) and is present in EATRO 164 cells. Alternative editing of the $3^{\prime}$ UTR by this gRNA (Fig. 3G[2]), as proposed by Koslowsky et al. (2013), allowed almost full annealing of the gRNA. This gRNA was almost identical in both strains (Fig. 3G[3]). Koslowsky et al. reported a second initiating gRNA gA6(770-822), a low abundance transcript not detected in our libraries.

\section{Native $\mathrm{H} 2$ and 3010 MRBs specifically copurify with pre-edited and edited mRNAs}

MRB1 complexes are only known to associate with gRNA (Weng et al. 2008; Hashimi et al. 2009; Hernandez et al. 2010). Because protein subunits of our immunopurified MRB samples including $\mathrm{H} 2$ photo-crosslinked with a synthetic mRNA (Fig. 1,E,F), we considered that H2-MRB, but possibly also 3010-MRB, purifications could contain endogenous mRNAs. Mitochondrial mRNAs may associate with
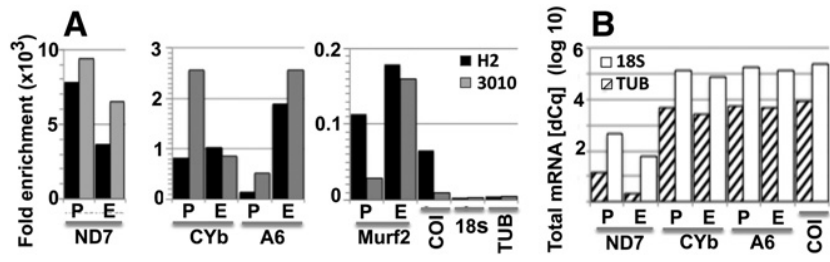

FIGURE 4. H2-MRB and 3010-MRB copurify with pre-edited and edited mRNAs. (A) Fold-enrichment in IPs of the samples normalized to the mock IP in qPCR assays. (E) Edited, (P) pre-edited (P), (COI) neveredited COI mRNA, two reference transcripts: (TUB) tubulin mRNA and (18S) nuclear $18 \mathrm{~S}$ rRNA (Fold $=2^{-\mathrm{ddCq}}$, where $\mathrm{ddCq}=\mathrm{Cq}$ test IP -Cq mock IP). (B) dCq of steady-state mRNAs in mitochondrial extract relative to background tubulin mRNA or nuclear $18 \mathrm{~S}$ rRNA used as reference $\left(\mathrm{dCq}=2^{\text {(Target } \mathrm{Cq}-\mathrm{Ref} \mathrm{Cq})}\right)$. For example, edited ND7 is more abundant than other edited mRNAs (i.e., has a lower Cq). All end-point amplicons in this study were single products during linear amplification, gel-purified and sequenced. Cq duplicates $($ STDEV $\leq 0.1)$ were averaged, and dilutions were adjusted to $100 \%$.

MRBs or with transiently bound factors including RECC or mitoribosomes (Weng et al. 2008; Hernandez et al. 2010; Aphasizheva et al. 2011). Initial primer extension assays of purified H2-MRB and 3010-MRB detected some mRNA ND7 but no other mRNAs (data not shown). Subsequent qPCR assays of purified MRBs and a mock IP revealed a robust enrichment of edited and pre-edited mRNAs (ND7, $\mathrm{CYb}, \mathrm{A} 6$, and Murf2) relative to never-edited mRNA (COI), nuclear 18S rRNA, and cytosolic tubulin (Fig. 4A). Housekeeping transcripts, usually carried over during purification, served as a reference in our assays. Because editing progresses in a $3^{\prime}-5^{\prime}$ direction and the amplicons $(\sim 50-100$ bp) score $5^{\prime}$ edited (or pre-edited) targets, the corresponding downstream sequences are presumed to be correctly edited. Interestingly, both the relative abundance and ratios of edited and pre-edited targets differ substantially between MRBs. Also, while all tested edited and pre-edited mRNAs were significantly more enriched than COI, the trend appeared somewhat more consistent in edited transcripts. Relative to the mock IP, edited ND7 was enriched several thousandfold, while edited $\mathrm{CYb}$ was enriched only about a hundredfold. In contrast, $\mathrm{COI}$ (never-edited) and $\mathrm{CYb}$ and A6 mRNAs (edited and pre-edited) exhibit comparable steady-state levels (Fig. 4B). Although, edited ND7 was about one-thousandfold more abundant than other mRNAs at steady-state (i.e., it exhibits the lowest $\mathrm{dCq}$ ), this difference was still several times smaller than the enrichment fold of edited ND7 in the

FIGURE 3. gRNA alignments at editing blocks B1 and B2 with currently annotated edited mRNAs, and predicted alternative editing patterns. Basepaired gRNA sequences are in $3^{\prime} \rightarrow 5^{\prime}$ orientation. Regions of interest are identified as follows: editing domain (bold), mature mRNA sequence (underlined), gRNA guide domain (gray box), length of the $3^{\prime} \mathrm{U}$ tail (subscript), and stop codon (double line). Proposed alternative U-insertions and Udeletions (" $\mathrm{t}$ " and “ $\star$ ", respectively, in a box) result in higher quality duplexes, i.e., longer guide domains and changes in encoded C-terminal amino acids (in ND7 $3^{\prime}$ domain $[B]$ and RPS12 $[F]$ ) or $3^{\prime}$ UTR (in CO3 $[D]$, ND8 $[E]$, and A6 $[G]$ ). No alternative editing is predicted for CyB $(A)$ and ND7 $5^{\prime}$ domain $(C)$. Homology alignments of gRNAs are given in $5^{\prime}-3^{\prime}$ orientation with identities (dotted boxes) and guide domains (gray boxes). Aligned gRNAs from the study in EATRO 164 cells by Koslowsky et al. (2013) are indicated. The gRNA numbering uses standard nomenclature indicating paired nucleotide positions in edited mRNAs. The arrow in ND8 indicates an extra guiding "A" in B1.alt. The arrow in RPS12 indicates a position in the guide domain of gRPS12(267-322) that forms an A.C mismatch with mRNA. 
3010-MRB sample. Should the mRNAs described above be largely bound to mitoribosomes contaminating our samples, never-edited mRNAs would be enriched, and pre-edited mRNAs should not be present (Aphasizheva et al. 2011). Altogether, these data suggest a selective copurification of MRBs with mRNAs that undergo editing, relative to neveredited COI and housekeeping transcripts.

\section{DISCUSSION}

MRB1 complexes, isolated via epitope tags and specific antibodies, exhibit overlapping protein composition that has been discussed (Hernandez et al. 2010; Ammerman et al. 2011, 2012; Cruz-Reyes and Read 2013; Hashimi et al. 2013). However, the RNA component of these particles was unknown. Our current study of native MRBs with different protein composition indicated that these particles also differ in RNA composition. We distinguished two MRBs that contained either REH2 or MRB3010 subunits. Based on this work and our previous study (Hernandez et al. 2010), we propose that REH2 forms an MRB subcomplex that contains gRNA, unwinding activity, and several subunits that can be photo-crosslinked to RNA. REH2-associated unwinding may remodel RNA-protein interactions needed for efficient editing substrate association with REH2-MRB (Hernandez et al. 2010; V Kumar, B Madina, and J Cruz-Reyes, unpubl.). Whether or not this activity affects editing substrate binding to other MRBs needs to be examined. REH2-dependent unwinding could also control global intra-strand mRNA structure or gRNA exchange during editing progression, as it was proposed for TbRGG2 and REH1, respectively (Ammerman et al. 2010; Li et al. 2011). It was proposed that RNAi-repression of REH2 reduces gRNA stability (Hashimi et al. 2009). We also saw some gRNA reduction at Day 6 of REH2 repression (Hernandez et al. 2010 and Fig. 5D therein). However, this may be an indirect result of the late time point or a small but specific impact on metabolically stable gRNA. Revised analyses by us and by another lab indicate that robust REH2 depletion at Day 4 of RNAi does not significantly impact the gRNA steady-state level (Supplemental Fig. S4; R Aphasizhev, pers. comm.). MRB3010 may not bind gRNA but affects an unidentified early editing step (Ammerman et al. 2011). The REH2 and 3010 subcomplexes examined here contain the core GAP1 subunit. GAP1/2 homologs are known to bind and stabilize gRNA (Weng et al. 2008). Interestingly, native REH2-MRB and 3010-MRB may share multiple RNA crosslinking subunits, suggesting a common RNA-binding core. So, REH2-MRB, 3010-MRB, and other MRB subcomplexes may transiently associate in higher-order MRB1 complexes that assemble editing substrates and RECCs creating an editing holoenzyme (Fig. 5; Simpson et al. 2010; Cruz-Reyes and Read 2013; Hashimi et al. 2013), and dynamic contacts between the individual holoenzyme components may modulate the editing process, as well as productive interactions with mitoribosomes and other mitochondrial factors.

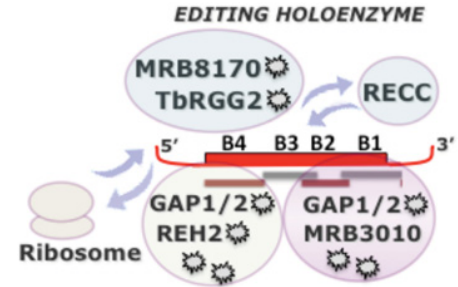

FIGURE 5. Working model. Dynamic higher-order MRB1 "organizers" composed of several specialized subcomplexes (MRBs) with different RNA/protein composition may coordinate recruitment of editing substrates (pre-edited mRNA and gRNA) and catalytic RECCs forming an editing holoenzyme. MRBs may bind edited mRNAs and route them into ribosomes by undefined "hand-over" mechanisms. Native helicase REH2 binds RNA, presumably gRNA, mRNA, or both, and forms a stable MRB with editing substrates, unwinding activity, and several subunits including other RNA-binding proteins (stars). REH2-associated unwinding seems to promote stable assembly of editing substrates with REH2-MRB but could also affect other MRBs and editing progression. The MRB3010 subunit affects an early editing step and may not bind RNA directly but forms a separate stable MRB. The current study showed that the MRB3010-subcomplex associates with editing substrates and edited mRNA and suggests that it promotes early editing through its preferential recruitment of initiating gRNAs. Both purified REH2-MRB and MRB3010-MRB contain GAP1/2 subunits that bind and stabilize gRNA. A common RNA-binding core in MRBs may include GAP proteins and several RNA crosslinking subunits that we detected but need to be identified. Dynamic interactions between these MRBs and additional factors (MRBs and non-MRBs not shown in the cartoon) may control substrate recruitment and interactions during initiation and progression between blocks (e.g., B1-to-B4). A proposed TbRGG2 MRB-like subcomplex does not contain REH2, MRB3010, or GAP proteins (Madina et al. 2011; Ammerman et al. 2012). Distinct RNA/protein composition of MRBs may also impact transient associations with RECCs, mitoribosomes, and other mitochondrial factors. Recombinant proteins known to crosslink with synthetic RNA (stars) include the following: GAPs with gRNA, and the 4160/8160 paralogs and TbRGG2 preferentially with mRNA-like fragments. The initiation gRNA anchor anneals just $3^{\prime}$ of the editing domain. Later anchors often need edited sequence. Whether gRNAs remain annealed to edited mRNA is unknown.

This study introduces NFPs (nucleotide frequency plots) of gRNAs, a simple but powerful tool that allows direct quantitative comparisons of gRNA content in purified complexes and total mitochondrial RNA. These NFP analyses provide (1) annotation of specific gRNAs at base resolution within editing domains, and (2) a measure of cumulative guide RNA potential at single and overlapping editing blocks in the parasite and purified complexes. This information is important because multiple redundant gRNA genes from polymorphic or entirely different minicircles often contribute to editing of the same block. Consistent with a role of the MRB3010 subunit in editing initiation, our NGS study of the first editing blocks in several mRNAs indicated a higher accumulation of initiating gRNAs in 3010-MRB than in H2-MRB. We note that similar amounts of gRNA (scored by capping of $5^{\prime}$ triphosphate ends) from IPs of REH 2 and MRB3010 were used in the construction of Illumina libraries. This was largely confirmed given that counts go up in some gRNAs and down in others between the libraries. It is 
possible that MRB3010 promotes specific recruitment or retention of accumulated initiating gRNAs in its subcomplex. A selective accumulation of initiating gRNA was especially clear for ND7 and ND8 but was also clear for other mRNAs. A partitioning of initiating gRNAs between MRBs, together with the observed low steady-state level of several initiating gRNAs in the parasite, both in our study and a recent report (Koslowsky et al. 2013), suggest that recruitment or usage of these particular gRNAs is controlled in vivo. Currently, we are working to determine the impact of initiating gRNA enrichment on the target mRNAs. Interestingly, the study by Koslowsky did not find one or more gRNAs for half the mRNA substrates, implying that rare gRNAs may stall editing progression.

Purified MRBs have only been reported to contain gRNA; however, we found between ten- and several thousand-fold specific enrichment of edited and pre-edited mRNAs in immunoselected MRBs relative to a mock IP. Housekeeping $18 \mathrm{~S}$ rRNA and tubulin mRNA were not enriched, and the never-edited mRNA COI may be slightly increased in the H2-MRB sample. MRB subcomplexes may specifically bind edited and pre-edited mRNAs in addition to gRNA, because RECCs are substoichiometric in purifications of MRB1 (Fig. 1B; Fisk et al. 2008; Weng et al. 2008; Hernandez et al. 2010), and natively purified RECC was reported to be largely devoid of RNA (Rusche et al. 1997). In contrast to our purifications of native MRBs, mitoribosomes associate with never-edited, but not with pre-edited, mRNAs (Aphasizheva et al. 2011). So, assembled MRB1 complexes may store and route edited mRNAs from RECCs into ribosomes (Fig. 5).

REH2 and the GAP1/2 homologs are the only known RNA-crosslinking subunits in the context of purified MRBs, but other RNA-binding proteins and their cognate RNA targets in vivo need to be identified. The mRNA probe in our crosslinking assays may or may not reflect the cognate target's specificity (Hernandez et al. 2010), and REH2 may potentially bind gRNA, mRNA, or both. Recombinant versions of TbRGG2 and the paralogs MRB8170 and MRB4160 were reported to preferentially crosslink in vitro with synthetic sequences resembling cognate mitochondrial mRNA (Fisk et al. 2008; Kafkova et al. 2012). Strong GAP1/2-dependent stability of gRNA in vivo and preferential crosslinking of a synthetic gRNA with GAPs in their purified MRB point to these transcripts as their natural target (Weng et al. 2008). The common $\sim 100-\mathrm{kDa}$ crosslink in native $\mathrm{H} 2$ $\mathrm{MRB}$ and 3010-MRB in our assays (Fig. 2A) may be induced by MRB8170, its paralog MRB4160, or both.

Several of the gRNAs, aligned to annotated mRNAs from early studies, predict alternative $3^{\prime}$ editing patterns within 3' UTR or ORF regions that could impact mRNA stability, translation efficiency, or the encoded C-terminal amino acid sequence. Notably, our study in Lister 427 procyclic cells and recent analyses in a different strain (EATRO 164 cells) indicate important differences in minicircle content or expression, including strain-specific gRNAs, e.g., gRNAs detected in our study but not in Koslowsky's. Continuing analysis of mRNA $3^{\prime}$ ends in our lab is consistent with our proposed alternative $3^{\prime}$ editing between strains (B Madina, V Kumar, and J Cruz-Reyes, unpubl.). The library of total gRNA examined by these authors achieved significantly higher depth than ours, as our preparations were limited by the gRNA amount extracted from purified MRBs. A high evolution rate of minicircles may create some variability in mitochondrial function among strains and thereby add adaptive potential in T. brucei. However, essential edits may be under strong selection pressure, as is illustrated by CYb gRNA genes in the same EATRO 164 cells used recently and in the early 1990s (Koslowsky et al. 1992, 2013) that have identical guide sequence but highly diverged nonguiding termini.

\section{MATERIALS AND METHODS}

\section{Cell culture}

T. brucei Lister strain 427 29-13 procyclic "PF" (tryps.rockefeller. edu) was grown axenically in log phase in SDM79 and harvested at a cell density of $1-3 \times 10^{7}$ cells/mL. A derived cell line expressing tetracycline-inducible TAP-REH2 was used as described (Hernandez et al. 2010).

\section{Protein purification}

Native MRB1 subcomplexes were immunoprecipitated from freshly prepared mitochondrial extracts (Harris and Hajduk 1992) using affinity-purified peptide antibodies raised in rabbit against REH2, MRB3010, and cytochrome oxidase 2 (mock) in T. brucei (Bethyl Laboratories, Inc), as we reported, with some changes (Hernandez et al. 2010). Briefly, specific antibodies of identical quality and concentration were conjugated to Protein A-Dynabeads (Life Technologies) that were pretreated with 5\% BSA. Approximately $2 \mathrm{mg}$ of mitochondrial extract was supplemented with $1 \times$ cOmplete Protease Inhibitor cocktail (Roche), SUPERase.In RNase inhibitor (Life Technologies), and precleared by passage over Protein A-Sepharose beads (GE Healthcare) before loading onto antibody-conjugated beads. Ectopically expressed TAP-REH2 was specifically immunopurified using IgG-Dynabeads (Life Technologies) as reported (Hernandez et al. 2010). All washes were performed at $200 \mathrm{mM}$ $\mathrm{NaCl}, 1 \mathrm{mM}$ EDTA, $10 \mathrm{mM} \mathrm{MgCl} 2$ and $25 \mathrm{mM}$ Tris, $\mathrm{pH} 8$.

\section{Western analyses and radioactive assays}

Western blots were examined with rabbit affinity-purified antiREH2 (Hernandez et al. 2010) and anti-MRB3010 polysera, or rabbit anti-GAP1 prebleed polyserum. Anti-MRB3010 antibodies were raised against the C-terminal peptide CPPLYQLYISRGSTPQA (Bethyl Laboratories, Inc), which is uniquely aligned to this protein in a Blast search of T. brucei. Monoclonal antibodies against REL1 ligase (a RECC subunit) were used as reported (Panigrahi et al. 2001). A 61-nt fragment of pre-edited A6 mRNA bearing a photoreactive 4thio- $\mathrm{U}$ and ${ }^{32} \mathrm{P}$ at the first editing site was mixed with immunopurified MRBs and subjected to 365-nm UV irradiation on ice, as previously described (Sacharidou et al. 2006; Cruz-Reyes 2007). Radioactive capping of gRNA and adenylation of RECC 
ligases were performed as reported (Blum et al. 1990; Sabatini and Hajduk 1995). Northern blots of RNA extracted from IPs from whole-cell lysate with 5' labeled probes for gA6 B1.alt [1357]: CCACTGTAAAACTGATTTCGTCATTGGAG (Tm 57.9) and gND7 3' domain B1 [1358]: CTTATACATGAAGTCACTGTAGG ATTG (Tm 53.3) were performed in ULTRAhyb solution (Life Technologies) and $2 \times$ SSC washes at $40^{\circ} \mathrm{C}$.

\section{Illumina libraries of gRNA from purified MRBs and procyclic parasites}

Identical immunoprecipitations were performed in parallel using REH2, 3010, and mock affinity-purified antibodies. The final washed beads were treated with proteinase K and SDS, and the associated RNA was treated with a DNA-free DNase kit and recovered by acid phenol-chloroform extraction and ethanol precipitation. RNA samples were treated with the ScriptCap capping system (CELLSCRIPT) and $\left[\alpha^{-32} \mathrm{P}\right]-\mathrm{GTP}$, and resolved on $15 \%$ UREAPAGE to concentrate the gRNA in a narrow band. Size markers of gRNA mobility ( $\sim 40-60 \mathrm{nt})$ were prepared with synthetic RNA fragments. Capped gRNA from IPs and total mitochondrial RNA were resolved in parallel with identical uncapped (unlabeled) samples (Supplemental Fig. S3). The labeled samples served as controls to adjust the amount of unlabeled gRNA used for library construction. We note that the total RNA sample was treated with Terminator 5' Phosphate-Dependent Exonuclease (Epicentre) that degrades rRNA but not gRNA, as these transcripts carry a $5^{\prime}$ triphosphate (Blum et al. 1990). This step was not applied to the IPs because much nuclear rRNA and cytosolic mRNA were selected out in those samples (Fig. 4A). Terminator-treated RNA was diluted to generate comparable amounts of labeled gRNA in the IP and total RNA lanes on the gel for concurrent excision of identical cuts, elution, and adapter ligations. We used an Illumina small RNA protocol with modifications. The IP and total gRNA samples were ligated to different barcoded $3^{\prime}$ adapters. At this point, all samples were mixed, and the subsequent steps were performed in a single reaction: phosphatase/PNK treatment to add a single phosphate, $5^{\prime}$ adapter ligation, cDNA synthesis, and PCR amplification. Analysis in an Agilent Bioanalyzer showed a narrow distribution of the libraries, with an average size of $\sim 150 \mathrm{bp}$, reflecting the average size of gRNA plus adapters. The multiplexed libraries were sequenced on Illumina GAIIx (single-end, 75-base run), producing $\sim 40$ million total reads.

\section{Computational analyses. Read preprocessing}

All base calls in the sequencing reads with a Phred score below 20 were converted into N's and runs of trailing N's were removed from the reads. Reads in which more than 10 N's remained were discarded. Sequencing adapters were identified by requiring at least the first five bases of the adapter to be contained in the read and the sequence identity between the known adapter sequence and the part of the read containing the adapter to be at least $75 \%$. Reads in which no adapter could be identified and reads with less than 10 bases after adapter removal were discarded. The bases immediately preceding the adapter were compared with the barcodes ATAC, ACCAA, CGAGA, and ATAGC, allowing at most one mismatch to distinguish reads originating from the procyclic, 3010, H2, and cytochrome oxidase 2 (mock) libraries, respectively, and the barcodes were removed. All reads with more than one mismatch to one of the four barcodes at the $3^{\prime}$ end were discarded.

\section{Alignment to edited mRNAs}

Reads from each library were separately aligned to the known edited mRNA sequences deposited at the KISS bioinformatics site (http:// splicer.unibe.ch/kiss/). To this end, all reads with three or more consecutive N's were discarded. Gapless semilocal alignments with a match score (including GU base-pairing) of 1 and a mismatch score of -2 were used to identify regions of local similarity between the reads and edited mRNA sequences. Reads with a local similarity score of at least 20 were parsed into a $5^{\prime}$ unmatched region, the matching region, a $3^{\prime}$ unmatched region, and a poly- $U$ tail. These reads were post-filtered in order to only retain reads with at most two mismatches, a minimum length of the matching region of 25 bases, and a poly-U tail of at least three U's. For RPS12, we also required that the length of the $5^{\prime}$ unmatched region was, at most, 20 bases. For the remaining reads, we tallied the number of bases in matching regions covering each position in the edited mRNA.

\section{Quantitative real-time PCR (qPCR)}

RNA from the IPs and procyclic cells used in the preparation of Illumina libraries was used for cDNA synthesis, after treatment with a DNA-free DNase kit, in reactions with random hexamers in the iScript cDNA kit (Bio-Rad). Twenty microliters qPCR reactions were performed with reported primers specific for edited or pre-edited mRNAs, never-edited, and two reference transcripts, $18 \mathrm{~S}$ rRNA and tubulin (Carnes and Stuart 2007), in a SsoFast EvaGreen Supermix (Bio-Rad). Diluted samples to score test transcripts (1:7) and reference transcripts (1:50) produced a single amplicon during linear amplification. All end-point amplicons described here were gel-isolated, cloned, and confirmed by sequencing. Fold-enrichment of mitochondrial and reference transcripts in the sample IPs, relative to the mock IP, was calculated as follows: Fold $=2^{(-\mathrm{ddCq})}$, where $\mathrm{ddCq}=\mathrm{Cq}$ test IP $-\mathrm{Cq}$ mock IP. Steadystate mRNAs in mitochondrial extract relative to background tubulin and nuclear 18s rRNA (reference transcripts) were scored as follows: $\mathrm{dCq}=2^{\text {(Target } \mathrm{Cq}-\text { Ref } \mathrm{Cq})} . \mathrm{Cq}$ duplicates of each sample (STDEV $\leq 0.1)$ were averaged, and dilutions were adjusted to $100 \%$.

\section{DATA DEPOSITION}

Deep sequencing data have been deposited in NCBI's Sequence Read Archive under accession number SAMN02795843.

\section{SUPPLEMENTAL MATERIAL}

Supplemental material is available for this article.

\section{ACKNOWLEDGMENTS}

We thank Dr. Melinda Wales for reading this manuscript, Ken Stuart for supplying monoclonal antisera against RECC subunits, and Laurie K. Read for her gift of polyclonal antiserum against GAP1. We also thank the Genomics and Bioinformatics Facility at TAMU for their support. This material is based upon work supported by the National Science Foundation under Grants No. 
NSF1122109 (J.C-R.) and NSF1105458 (R.B.). This work was also supported in part by National Institutes of Health, NIH R01 AI088011 (B.H.M.M.)

Received February 2, 2014; accepted April 25, 2014.

\section{REFERENCES}

Alatortsev VS, Cruz-Reyes J, Zhelonkina AG, Sollner-Webb B. 2008. Trypanosoma brucei RNA editing: Coupled cycles of U deletion reveal processive activity of the editing complex. Mol Cell Biol 28: 2437-2445.

Ammerman ML, Presnyak V, Fisk JC, Foda BM, Read LK. 2010. TbRGG2 facilitates kinetoplastid RNA editing initiation and progression past intrinsic pause sites. RNA 16: 2239-2251.

Ammerman ML, Hashimi H, Novotna L, Cicova Z, McEvoy SM, Lukes J, Read LK. 2011. MRB3010 is a core component of the MRB1 complex that facilitates an early step of the kinetoplastid RNA editing process. RNA 17: 865-877.

Ammerman ML, Downey KM, Hashimi H, Fisk JC, Tomasello DL, Faktorova D, Kafkova L, King T, Lukes J, Read LK. 2012. Architecture of the trypanosome RNA editing accessory complex, MRB1. Nucleic Acids Res 40: 5637-5650.

Aphasizhev R, Aphasizheva I. 2011. Mitochondrial RNA processing in trypanosomes. Res Microbiol 162: 655-663.

Aphasizheva I, Maslov D, Wang X, Huang L, Aphasizhev R. 2011. Pentatricopeptide repeat proteins stimulate mRNA adenylation/uridylation to activate mitochondrial translation in trypanosomes. Mol Cell 42: 106-117.

Blum B, Simpson L. 1990. Guide RNAs in kinetoplastid mitochondria have a nonencoded $3^{\prime}$ oligo(U) tail involved in recognition of the preedited region. Cell 62: 391-397.

Blum B, Bakalara N, Simpson L. 1990. A model for RNA editing in kinetoplastid mitochondria: "Guide" RNA molecules transcribed from maxicircle DNA provide the edited information. Cell 60: 189-198.

Carnes J, Stuart KD. 2007. Uridine insertion/deletion editing activities. Methods Enzymol 424: 25-54.

Carnes J, Soares CZ, Wickham C, Stuart K. 2011. Endonuclease associations with three distinct editosomes in Trypanosoma brucei. J Biol Chem 286: 19320-19330.

Cifuentes-Rojas C, Halbig K, Sacharidou A, De Nova-Ocampo M, CruzReyes J. 2005. Minimal pre-mRNA substrates with natural and converted sites for full-round $U$ insertion and $U$ deletion RNA editing in trypanosomes. Nucleic Acids Res 33: 6610-6620.

Cruz-Reyes J. 2007. RNA-protein interactions in assembled editing complexes in trypanosomes. Methods Enzymol 424: 107-125.

Cruz-Reyes J, Read LK. 2013. Coordination of RNA editing with other RNA processes in kinetoplastid mitochondria. In RNA editing: current research and future trends (ed. Maas S), pp. 65-90. Caister Academic Press, Norfolk, United Kingdom.

Cruz-Reyes J, Zhelonkina A, Rusche L, Sollner-Webb B. 2001. Trypanosome RNA editing: simple guide RNA features enhance U deletion 100-fold. Mol Cell Biol 21: 884-892.

Fisk JC, Ammerman ML, Presnyak V, Read LK. 2008. TbRGG2, an essential RNA editing accessory factor in two Trypanosoma brucei life cycle stages. J Biol Chem 283: 23016-23025.

Harris ME, Hajduk SL. 1992. Kinetoplastid RNA editing: in vitro formation of cytochrome b gRNA-mRNA chimeras from synthetic substrate RNAs. Cell 68: 1091-1099.

Hashimi H, Cicova Z, Novotna L, Wen YZ, Lukes J. 2009. Kinetoplastid guide RNA biogenesis is dependent on subunits of the mitochondrial RNA binding complex 1 and mitochondrial RNA polymerase. RNA 15: 588-599.

Hashimi H, Zimmer SL, Ammerman ML, Read LK, Lukes J. 2013. Dual core processing: MRB1 is an emerging kinetoplast RNA editing complex. Trends Parasitol 29: 91-99.
Hernandez A, Madina BR, Ro K, Wohlschlegel JA, Willard B, Kinter MT, Cruz-Reyes J. 2010. REH2 RNA helicase in kinetoplastid mitochondria: ribonucleoprotein complexes and essential motifs for unwinding and guide RNA (gRNA) binding. J Biol Chem 285: $1220-1228$

Kafkova L, Ammerman ML, Faktorova D, Fisk JC, Zimmer SL, Sobotka R, Read LK, Lukes J, Hashimi H. 2012. Functional characterization of two paralogs that are novel RNA binding proteins influencing mitochondrial transcripts of Trypanosoma brucei. RNA 18: 1846-1861.

Koslowsky DJ, Riley GR, Feagin JE, Stuart K. 1992. Guide RNAs for transcripts with developmentally regulated RNA editing are present in both life cycle stages of Trypanosoma brucei. Mol Cell Biol 12: 2043-2049.

Koslowsky D, Sun Y, Hindenach J, Theisen T, Lucas J. 2013. The insectphase gRNA transcriptome in Trypanosoma brucei. Nucleic Acids Res 42: $1873-1886$

Li F, Herrera J, Zhou S, Maslov DA, Simpson L. 2011. Trypanosome REH1 is an RNA helicase involved with the $3^{\prime}-5^{\prime}$ polarity of multiple gRNA-guided uridine insertion/deletion RNA editing. Proc Natl Acad Sci 108: 3542-3547.

Madina BR, Kuppan G, Vashisht AA, Liang YH, Downey KM, Wohlschlegel JA, Ji X, Sze SH, Sacchettini JC, Read LK, et al. 2011. Guide RNA biogenesis involves a novel RNase III family endoribonuclease in Trypanosoma brucei. RNA 17: 1821-1830.

Madison-Antenucci S, Grams J, Hajduk SL. 2002. Editing machines: the complexities of trypanosome RNA editing. Cell 108: 435-438.

Ochsenreiter T, Cipriano M, Hajduk SL. 2007. KISS: The kinetoplastid RNA editing sequence search tool. RNA 13: 1-4.

Ochsenreiter T, Anderson S, Wood ZA, Hajduk SL. 2008. Alternative RNA editing produces a novel protein involved in mitochondrial DNA maintenance in trypanosomes. Mol Cell Biol 28: 5595-5604.

Panigrahi AK, Schnaufer A, Carmean N, Igo RP Jr, Gygi SP, Ernst NL, Palazzo SS, Weston DS, Aebersold R, Salavati R, et al. 2001. Four related proteins of the Trypanosoma brucei RNA editing complex. Mol Cell Biol 21: 6833-6840.

Panigrahi AK, Zikova A, Dalley RA, Acestor N, Ogata Y, Anupama A, Myler PJ, Stuart KD. 2008. Mitochondrial complexes in Trypanosoma brucei: a novel complex and a unique oxidoreductase complex. Mol Cell Proteomics 7: 534-545.

Rusche LN, Cruz-Reyes J, Piller KJ, Sollner-Webb B. 1997. Purification of a functional enzymatic editing complex from Trypanosoma brucei mitochondria. EMBO J 16: 4069-4081.

Sabatini R, Hajduk SL. 1995. RNA ligase and its involvement in guide RNA/mRNA chimera formation. Evidence for a cleavage-ligation mechanism of Trypanosoma brucei mRNA editing. J Biol Chem 270: 7233-7240

Sacharidou A, Cifuentes-Rojas C, Halbig K, Hernandez A, Dangott LJ, De Nova-Ocampo M, Cruz-Reyes J. 2006. RNA editing complex interactions with a site for full-round U deletion in Trypanosoma brucei. RNA 12: 1219-1228.

Simpson L, Thiemann OH, Savill NJ, Alfonzo JD, Maslov DA. 2000. Evolution of RNA editing in trypanosome mitochondria. Proc Natl Acad Sci 97: 6986-6993.

Simpson L, Aphasizhev R, Gao G, Kang X. 2004. Mitochondrial proteins and complexes in Leishmania and Trypanosoma involved in U-insertion/deletion RNA editing. RNA 10: 159-170.

Simpson L, Aphasizhev R, Lukes J, Cruz-Reyes J. 2010. Guide to the nomenclature of kinetoplastid RNA editing: a proposal. Protist 161: 2-6.

Stuart K, Allen TE, Kable ML, Lawson S. 1997. Kinetoplastid RNA editing: complexes and catalysts. Curr Opin Chem Biol 1: 340-346.

Vickerman K. 1985. Developmental cycles and biology of pathogenic trypanosomes. Br Med Bull 41: 105-114.

Weng J, Aphasizheva I, Etheridge RD, Huang L, Wang X, Falick AM, Aphasizhev R. 2008. Guide RNA-binding complex from mitochondria of trypanosomatids. Mol Cell 32: 198-209. 

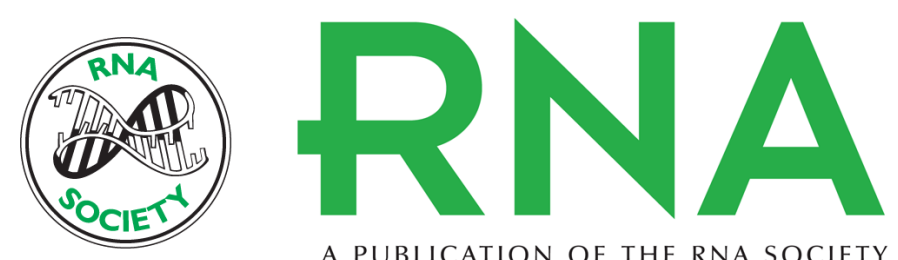

A PUBLICATION OF THE RNA SOCIETY

\section{Native mitochondrial RNA-binding complexes in kinetoplastid RNA editing differ in guide RNA composition}

Bhaskara R. Madina, Vikas Kumar, Richard Metz, et al.

RNA 2014 20: 1142-1152 originally published online May 27, 2014

Access the most recent version at doi:10.1261/rna.044495.114

\section{Supplemental http://rnajournal.cshlp.org/content/suppl/2014/05/20/rna.044495.114.DC1 \\ Material}

References This article cites 37 articles, 21 of which can be accessed free at: http://rnajournal.cshlp.org/content/20/7/1142.full.html\#ref-list-1

Creative This article is distributed exclusively by the RNA Society for the first 12 months after the Commons

License full-issue publication date (see http://rnajournal.cshlp.org/site/misc/terms.xhtml). After 12 months, it is available under a Creative Commons License (Attribution-NonCommercial 4.0 International), as described at http://creativecommons.org/licenses/by-nc/4.0/. Email Alerting $\begin{aligned} & \text { Receive free email alerts when new articles cite this article - sign up in the box at the } \\ & \text { Service }\end{aligned}$ top right corner of the article or click here. 